\title{
PAPER
}

\section{White matter hyperintensities are related to physical disability and poor motor function}

\author{
P S Sachdev, W Wen, H Christensen, A F Jorm
}

J Neurol Neurosurg Psychiatry 2005;76:362-367. doi: 10.1136/jnnp.2004.042945

See end of article for authors' affiliations

\section{.................... \\ Correspondence to:}

Professor P Sachdev, NPI,

Prince of Wales Hospital, Barker Street, Randwick NSW 2031, Australia; p.sachdev@unsw.edu.au

Received 7 April 2004 In revised form 16 June 2004 Accepted 17 June 2004
Objective: To determine the impact of white matter hyperintensities (WMHs) on physical health and cognitive function in 60-64 year old individuals residing in the community.

Methods: A subsample of 478 persons aged 60-64 from a larger community sample underwent brain magnetic resonance imaging (MRI) scans. WMHs on T2 weighted FLAIR (fluid attenuated inversion recovery) MRI scans were assessed using an automated procedure. Subjects were assessed for global cognitive function, episodic memory, working memory (digit span), information processing speed (Symbol Digit Modalities Test; SDMT), fine motor dexterity (Purdue Pegboard), and grip strength, and completed the Physical Component Summary of the Short Form Health Survey (SF-12). Regression analyses were used to examine the effect of WMHs on physical and cognitive function.

Results: Deep and periventricular WMHs were present in all subjects, with women having slightly more lesions than men. WMHs were significantly associated with poorer reported physical health on the SF-12 scale, after adjusting for depression, cognitive function, and brain atrophy. WMHs were also related to lower scores on the Purdue Pegboard test, grip strength, choice reaction time, and SDMT, but not on tests of episodic memory, working memory, general intellectual function, and global cognitive function. On regression analyses, the Purdue Pegboard test and grip strength were related to physical disability.

Conclusion: WMHs are common, albeit mild, in middle adult life. They are associated with physical disability, possibly through reduced speed, fine motor coordination, and muscular strength. They are also related to slowed information processing speed but not other cognitive functions. t is common to detect white matter hyperintensities (WMHs) on $\mathrm{T} 2$ weighted magnetic resonances imaging (MRI) in the brains of clinically healthy older individuals. ${ }^{1}$ There is accumulating evidence that these incidental findings are not benign, but are associated with several impairments, such as cognitive deficits, ${ }^{2}$ gait and balance problems, urinary dysfunction, ${ }^{4}$ and depression. ${ }^{5}$ Although WMHs do not have a specific pathology, the lesions seen incidentally in elderly individuals with no history of neurological disorder are considered to be generally ischaemic in origin. ${ }^{6}$

Most research into WMHs has involved subjects who are elderly; that is, older than 65 years. However, these lesions are also seen commonly in individuals in their $50 \mathrm{~s}$ and $60 \mathrm{~s}$, especially those with risk factors for small vessel disease, such as hypertension and diabetes. ${ }^{7}$ The question of whether they produce impairment in mid-adult life has not been adequately investigated. It is possible that at an earlier age, because the load of white matter lesions is small, the brain has sufficient reserve capacity to overcome any dysfunction. The findings in older individuals suggest that the relation between WMHs and impairment increases with age, ${ }^{2}$ so that the examination of a population in mid-adult life is of interest.

In our study, we examined physical and cognitive associations of WMHs in a community sample of 60-64 year old individuals. The younger age of our population sample made it unlikely that clinically apparent gait and balance problems would be present. Therefore, the assessment included tests of fine motor speed and muscle strength, in addition to a subjective report of functional impairment.

\section{METHODS}

\section{Subjects}

The sample was drawn from the PATH Through Life Project designed to study the risk and protection factors for normal aging, dementia, and other neuropsychiatric disorders. The PATH Project cohort comprised 2551 individuals aged 60-64 years, who were residents of the city of Canberra and the adjacent town of Queanbeyan, Australia, and were recruited randomly through the electoral roll. Enrolment to vote is compulsory for Australian citizens. The response rate was $58.3 \%$ for the total sample-the cohort was obtained from an initial random mail contact of 4375 individuals. Subjects were asked at the initial interview if they would be willing to undergo an MRI scan. Those who were unwilling during the initial interview were significantly $(\mathrm{p}<0.0 .05)$ more likely to be women, of non-English speaking background, less well educated, with poorer physical health, and with lower cognitive test scores. Of those who had indicated willingness to undergo a scan, 622 were approached, and 478 (77\%) $(252$ men) provided written informed consent to undergo an MRI brain scan. Those who refused $(n=144)$ to undergo a scan when actually invited were significantly more likely to be of non-English speaking background, less well educated, with poorer physical health, and lower cognitive scores. Approval for our study was obtained from the human research ethics committees of the Australian National University, Canberra and the University of New South Wales, Sydney, Australia.

\section{MRI scans}

All subjects were imaged with a 1.5 Tesla Philips Gyroscan ACS-NT scanner (Philips Medical Systems, Best, the

Abbreviations: CSF, cerebrospinal fluid; CVLT, California Verbal Learning Test; $\mathrm{df}$, degrees of freedom; DWMH, deep white matter region; FLAIR, fluid attenuated inversion recovery; FOV, field of view; ICC, intraclass correlation; ICV, intracranial volume; MMSE, Mini Mental State Examination; MRI, magnetic resonance imaging; PVH, periventricular region; SDMT, Symbol Digits Modalities Test; SF-12, Physical Component Summary of the 12 ltem Short Form Health Survey; $T E$, echo time; TR, repetition time; WMH, white matter hyperintensity 


\begin{tabular}{|c|c|c|c|}
\hline Characteristic & Men $(n=250)$ & Women $(n=226)$ & $\mathrm{p}$ Value \\
\hline \multicolumn{4}{|l|}{ Sociodemographic factors } \\
\hline Age & $62.56(1.44)$ & $62.54(1.47)$ & 0.854 \\
\hline Years of education & $14.50(2.48)$ & $13.63(2.66)$ & $<0.001$ \\
\hline Married & $85.5 \%$ & $72.1 \%$ & $<0.001$ \\
\hline Employed full time & $30.6 \%$ & $14.6 \%$ & $<0.001$ \\
\hline White & $96.8 \%$ & $95.1 \%$ & 0.483 \\
\hline \multicolumn{4}{|l|}{ Health } \\
\hline Physical disability (SF-12)* & $50.74(7.82)$ & $48.95(9.54)$ & 0.026 \\
\hline History of heart disease & $14.1 \%$ & $8.4 \%$ & 0.060 \\
\hline History of stroke or TIA & $4.0 \%$ & $4.4 \%$ & 1.000 \\
\hline Diabetes & $2.3 \%$ & $0.5 \%$ & 0.217 \\
\hline PHQ depression score & $2.04(2.72)$ & $2.57(3.10)$ & 0.049 \\
\hline Hypertension & $61.7 \%$ & $46.5 \%$ & 0.005 \\
\hline Smoker & $7.3 \%$ & $8.0 \%$ & 0.863 \\
\hline Harmful/hazardous alcohol use & $6.5 \%$ & $4.9 \%$ & 0.553 \\
\hline \multicolumn{4}{|l|}{ Cognitive functioning } \\
\hline MMSE & $29.26(1.15)$ & $29.40(1.06)$ & 0.165 \\
\hline CVLT, immediate recall & $6.99(1.95)$ & $7.92(2.16)$ & $<0.001$ \\
\hline CVLT, delayed recall & $6.10(2.10)$ & $7.08(2.34)$ & $<0.001$ \\
\hline Symbol Digit Modalities Test & $50.98(8.25)$ & $51.07(9.00)$ & 0.905 \\
\hline Digits Backwards & $5.26(2.20)$ & $4.68(2.02)$ & 0.003 \\
\hline Spot the Word & $52.72(6.04)$ & $51.89(5.63)$ & 0.126 \\
\hline \multicolumn{4}{|l|}{ Motor functioning } \\
\hline Purdue Pegboard: both hands & $10.25(1.63)$ & $10.94(1.58)$ & $<0.001$ \\
\hline Purdue Pegboard: dominant hand & $12.92(1.92)$ & $14.01(1.92)$ & $<0.001$ \\
\hline Purdue Pegboard: non-dominant hand & $12.61(1.88)$ & $13.36(1.80)$ & $<0.001$ \\
\hline Simple reaction time (msec) & $241.6(43.1)$ & $258.2(57.6)$ & $<0.001$ \\
\hline Choice reaction time (msec) & $313.8(38.3)$ & $320.9(49.2)$ & 0.086 \\
\hline Grip strength (kg) & $42.76(6.86)$ & $26.08(5.46)$ & $<0.001$ \\
\hline
\end{tabular}

Netherlands) for Tl weighted three dimensional structural and T2 weighted fluid attenuated inversion recovery (FLAIR) sequence MRI. A scout midsagittal image was first acquired to locate the anterior to posterior commissure plane. The Tl weighted MRI was acquired in coronal orientation using a Tl-FFE sequence with the following parameters: repetition time $(\mathrm{TR}) / \mathrm{echo}$ time $(\mathrm{TE})=28.05 / 2.64 \mathrm{~ms}$; flip angle $=$ $30^{\circ}$; matrix size $=256 \times 256$; field of view $(\mathrm{FOV})=260 \times$ $260 \mathrm{~mm}$; slice thickness $=2.0 \mathrm{~mm}$, and mid-slice to midslice distance $=1.0 \mathrm{~mm}$, yielding over-contiguous coronal slices. The FLAIR sequence was acquired in coronal orientation with TR/TE/inversion time $=11000 / 140 / 2600$ ms; matrix size $=256 \times 256 ; \quad$ FOV $=230 \times 230 \mathrm{~mm} ; \quad$ slice thickness $=$ $4.0 \mathrm{~mm}$ with no gap between slices.

\section{Image analysis}

MRI scans were transferred to an independent Windows NT workstation and analysed using the software packages ANALYZE (Mayo Foundation, Rochester, Michigan, USA) and SPM99 (Cognitive Neuroscience Group, National Hospital for Nervous Diseases, London, UK). WMHs were identified on FLAIR sequences. A special computer program was written by one of us (WW) to delineate automatically WMHs in both the periventricular (PVH) and deep white matter (DWMH) regions. ${ }^{8}$ The absolute volumes of total white matter and WMHs were determined, and the percentage of white matter with a hyperintense signal was calculated for each subject. Twenty scans were processed twice to determine the re-test reliability of the procedure, and $100 \%$ agreement was noted. For concurrent validity, the scans were visually rated by two independent clinicians experienced in examining MRI scans on a modified Fazekas' scale. ${ }^{9}$ One hundred scans were visually rated and the intraclass correlation (ICC) was high for whole brain WMH (ICC, 0.43; $\mathrm{F}=1.76$; degrees of freedom $(\mathrm{df})=99$; $\mathrm{p}=0.003), \quad$ DWMH $\quad($ ICC, $0.63 ; \quad \mathrm{F}=2.74 ; \quad \mathrm{df}=99 ;$ $\mathrm{p}<0.005)$, and PVH (ICC, 0.59; $\mathrm{F}=2.44 ; \quad \mathrm{df}=99$; $\mathrm{p}<0.005)$. Pearson correlations were also carried out on a subset of the sample $(\mathrm{n}=100)$ and the results were: whole brain WMH, $r=0.791$ ( $\mathrm{p}<0.005)$; DWMH, $r=0.724$ $(\mathrm{p}<0.005)$; and PVH, $r=0.717(\mathrm{p}<0.005)$. To validate the automated method further, a trained operator manually outlined the WMHs in 25 brains. The agreement between the automated and manual methods was very high, with whole brain WMH scores for the two methods having an ICC of $0.998(p<0.0005)$ using the two way fixed effects model.

Using a standard atlas, ${ }^{10}$ we traced anatomical regions (DWMH: frontal, parietal, temporal, and occipital; PVH: anterior cap, posterior cap, and periventricular body) on the standard single brain included in the SPM99 software. Using these brain region of interest masks, the WMH volumes, number, location, and size were calculated automatically by implementing some of the tools provided by FSL (Image Analysis Group, FMRIB, Oxford University, UK: http:// www.fmrib.ox.ac.uk/fsl/) in the script language PERL. Because both linear and non-linear transforms were applied on to each individual MRI, the WMH thus measured did not equate exactly to the absolute volume. The relative valuethat is, the "density" (ratio of WMH against WM, expressed as a percentage) of the WMH of each individual is used.

The volumes of grey matter, white matter, and cerebrospinal fluid (CSF) were calculated after the segmentation of T1 weighted MRI using SPM99. Intracranial volume (ICV) was measured as the sum of the total grey matter, white matter, and CSF, and total brain volume as the sum of total grey and white matter. A brain atrophy index was calculated using the formula (ICV - brain volume)/ICV.

\section{Cognitive measures}

A brief battery of cognitive tests was administered to the subjects within three months of the MRI scan, which comprised the following: reaction time ("simple" measured 
over 80 trials, and "choice" measured over 40 trials), ${ }^{11}$ Digit Span, ${ }^{12}$ Symbol Digits Modalities Test (SDMT), ${ }^{13}$ California Verbal Learning Test (CVLT) (immediate recall and delayed recall after one trial), ${ }^{14}$ Spot the Word Test, ${ }^{15}$ and Mini Mental State Examination (MMSE). ${ }^{16}$ Current depression was measured using the depression section of the PRIME-MD Patient Health Questionnaire, which asks about DSM-IV Major Depression symptoms in the past two weeks. ${ }^{17}$ This questionnaire was scored to give a continuous scale from 0 to 27 .

\section{Physical health and motor system measures}

Physical disability was assessed using the Physical Component Summary of the 12 Item Short Form Health Survey (SF-12). ${ }^{18}$ This is a self report questionnaire that rates the subject's limitation in various activities (vigorous, such as running; moderate, such as vacuum cleaning or bowling; lifting groceries; climbing stairs; bending or kneeling; walking from $100 \mathrm{~m}$ to $>1 \mathrm{~km}$; bathing or dressing; performing regular work). The Purdue Pegboard test (both hands $)^{19}$ was used to measure fine motor dexterity, and grip strength was measured with a dynamometer. ${ }^{20}$

\section{Analysis of data}

Descriptive statistics on the sample were given as means and SDs for continuous variables and per cent prevalence for dichotomous variables. Sex differences in sample characteristics were evaluated using analysis of variance for the continuous variables and exact $\chi^{2}$ tests for the dichotomous variables. Linear regression analyses were performed to determine the contribution of WMHs to the variance in physical and cognitive variables. The total brain and regional WMH load, measured as a percentage of white matter affected, was used for these analyses. In the first instance, the analyses were adjusted for age, sex, and depression ratings. Because it is known that poor cognition can affect physical health, these analyses were repeated after adjusting for MMSE score and immediate recall score on the CVLT. Interactions between sex and WMHs were examined in the regression analyses but none were found and these are not reported here. Because a large number of regression analyses were carried out, the more conservative $p<0.01$ level was used to reduce type I errors.

\section{RESULTS}

Table 1 details the descriptive characteristics of the sample. Data were incomplete for two subjects.

\section{White matter hyperintensities}

WMHs were present in all 60-64 year old subjects, but their extent and distribution varied considerably (table 2 ). The mean (SD) volume of WMH was 4.904 (4.976) $\mathrm{ml} / \mathrm{subject}$, which represented $0.83 \%(0.84 \%)$ of the total white matter. Most WMHs (61.75\%) were in the periventricular region, with the anterior horn $(98.7 \%)$ being more likely to be affected than the posterior horn (91.8\%). WMHs were present in the deep white matter in 461 (96.6\%) subjects, with the WMHs spread throughout the cerebral hemispheres. The percentages of subjects with WMHs in different brain regions were as follows: frontal $(76.7 \%)$, temporal $(55.8 \%)$, parietal $(79.5 \%)$, occipital $(75.7 \%)$, cerebellum (1\%), and brain stem (1.9\%). However, the proportion of deep white matter affected was only $0.358 \%$, whereas the proportion of periventricular white matter affected was $4.35 \%(\mathrm{t}=28.10$; $\mathrm{df}=476 ; \mathrm{p}=0.000$, paired $t$ test).

The relative extent of WMHs, both deep and periventricular, was greater in women than in men, although the difference was small. The mean (SD) density of WMHs was $0.95 \%(1.04 \%)$ in women and $0.75 \%(0.63 \%)$ in men $(\mathrm{t}=-2.33 ; \mathrm{df}=446 ; \mathrm{p}=0.02)$. Women had higher WMH density in the periventricular region, the anterior and posterior caps, and the frontal, temporal, and parietal deep white matter ( $p<0.05$, uncorrected). The brain atrophy ratio ((intracranial volume - brain volume)/intracranial volume) was not different between men and women (0.21 for both).

\section{Cognitive deficits and WMHs}

The Spot the Word test ${ }^{15}$ score, as a measure of verbal intelligence, was not associated with WMHs. Regression analyses examined the relation between WMHs and immediate recall, delayed recall, the SDMT, and digit backwards, controlling for age, sex, and depression severity. There were no significant relations at the $\mathrm{p}<0.01$ level for either whole brain or regional WMH densities. However, choice reaction time was significantly related to WMHs. To remove the effect of motor slowing on choice reaction time, we performed regression analyses to determine the association between total and regional WMHs and choice reaction time, using simple reaction time as a covariate. The results, after adjusting for age, sex, and depression, were significant at the 0.01 level for total WMHs $(\mathrm{B}=6 ; \beta=0.12$; $\mathrm{p}=0.001)$, frontal WMHs $(\mathrm{B}=4, \beta=0.10 ; \mathrm{p}=0.008)$, occipital WMHs $(\mathrm{B}=6 ; \beta=0.10 ; \mathrm{p}=0.007)$, posterior horn WMHs $(\mathrm{B}=3 ; \beta=0.16 ; \mathrm{p}<0.001)$, and periventricular WMHs $(\mathrm{B}=1 ; \beta=0.10 ; \mathrm{p}=0.008)$.

\section{Physical health}

WMHs were significantly associated with the physical component score of the SF-12, with higher amounts of WMHs being related to poorer physical health (table 2). When WMHs in different brain regions were examined for this association, a significant relation was present for WMHs in frontal and parietal regions. The components of this scale were further examined for this relation. Those with increased WMHs rated their health as being poor, and stated that they were significantly more limited in both vigorous and moderate activities, irrespective of whether these were simple, such as walking and climbing stairs, or complex such as pushing a vacuum cleaner or playing bowls. This report of physical health was not accounted for by depression. We investigated whether brain atrophy rather than WMHs, cognitive impairment, or vascular risk factors such as hypertension and diabetes could account for the poorer physical health. We performed a further regression analysis predicting total WMHs that adjusted for hypertension, diabetes, the MMSE score, immediate recall on CVLT, and the brain atrophy index, and found very similar results (physical disability: $\mathrm{B}=-2.00 ; \quad \beta=-0.22 ; \quad \mathrm{p}<0.001$; Purdue Pegboard, dominant hand: $\mathrm{B}=-0.18 ; \beta=-0.08$; $\mathrm{p}=0.170$; Purdue Pegboard, non-dominant hand: $\mathrm{B}=-0.26$; $\beta=-0.12 ; \quad p=0.038 ; \quad$ Purdue Pegboard, both hands: $\mathrm{B}=-0.18 ; \beta=-0.09 ; \mathrm{p}=0.114 ;$ grip strength: $\mathrm{B}=-1.45$; $\beta=-0.13 ; p=0.001$; simple reaction time: $B=2 ; \beta=0.03$; $\mathrm{p}=0.583)$. Because it was hypothesised that WMHs were causing the impairment in physical health, mediating variables (fine motor movement on Pegboard, reaction time ( simple and complex), speed of information processing on SDMT, and grip strength) were examined. Both the Pegboard test score $(\mathrm{B}=0.82 ; \beta=0.16 ; \mathrm{p}=0.001)$ and grip strength $(\mathrm{B}=0.20 ; \beta=0.24 ; \mathrm{p}<0.0001)$ emerged as significant variables for this association.

\section{DISCUSSION}

Our study shows that WMHs are related to poor physical health. Subjects with high amounts of WMHs reported that their physical health limited them in walking short $(100 \mathrm{~m})$ and long $(>1 \mathrm{~km})$ distances, performing work related or other activities, participating in moderate level (for example, 
Table 2 Results of regression analyses predicting physical functioning variables from WMHs: unstandardised regression coefficients (B) and standardised coefficients ( $\beta$ ) adjusted for age, sex, and depressive symptoms

\begin{tabular}{|c|c|c|c|c|c|c|c|}
\hline WMH region & $\begin{array}{l}\text { SF-12 physical } \\
\text { component score }\end{array}$ & $\begin{array}{l}\text { Purdue } \\
\text { Pegboard (BH) }\end{array}$ & $\begin{array}{l}\text { Purdue } \\
\text { Pegboard (DH) }\end{array}$ & $\begin{array}{l}\text { Purdue } \\
\text { Pegboard (NDH) }\end{array}$ & $\begin{array}{l}\text { Simple reaction } \\
\text { time (msec) }\end{array}$ & $\begin{array}{l}\text { Choice } \\
\text { reaction time } \\
\text { (msec) }\end{array}$ & Grip strength $(\mathbf{k g})$ \\
\hline \multicolumn{8}{|l|}{ Frontal } \\
\hline B & -1.46 & -0.22 & -0.20 & -0.22 & 0 & 4 & -0.76 \\
\hline$\beta$ & -0.17 & -0.14 & -0.10 & -0.12 & 0.00 & 0.10 & -0.07 \\
\hline p Value & $<0.001$ & 0.002 & 0.019 & 0.007 & 0.939 & 0.028 & 0.008 \\
\hline \multicolumn{8}{|l|}{ Temporal } \\
\hline B & -3.77 & -0.72 & -1.27 & -1.14 & 14 & 21 & -3.29 \\
\hline$\beta$ & -0.10 & -0.10 & -0.15 & -0.14 & 0.06 & 0.11 & -0.07 \\
\hline p Value & 0.023 & 0.022 & 0.001 & 0.002 & 0.160 & 0.017 & 0.008 \\
\hline \multicolumn{8}{|l|}{ Parietal } \\
\hline B & -1.72 & -0.21 & -0.15 & -0.29 & 1 & 5 & -0.97 \\
\hline$\beta$ & -0.16 & -0.11 & -0.06 & -0.13 & 0.01 & 0.10 & -0.08 \\
\hline p Value & $<0.001$ & 0.017 & 0.159 & 0.004 & 0.826 & 0.037 & 0.007 \\
\hline \multicolumn{8}{|l|}{ Occipital } \\
\hline B & -0.24 & -0.06 & -0.01 & -0.03 & 9 & 10 & -0.61 \\
\hline$\beta$ & -0.02 & -0.03 & -0.00 & -0.01 & 0.13 & 0.18 & -0.04 \\
\hline $\mathrm{p}$ Value & 0.639 & 0.534 & 0.963 & 0.796 & 0.005 & 0.000 & 0.113 \\
\hline \multicolumn{8}{|l|}{ Cerebellum } \\
\hline B & -2.23 & -1.47 & -1.18 & -0.67 & 0 & 16 & 3.96 \\
\hline$\beta$ & -0.02 & -0.07 & -0.04 & -0.03 & 0.01 & 0.03 & 0.03 \\
\hline $\mathrm{p}$ Value & 0.662 & 0.129 & 0.306 & 0.546 & 0.858 & 0.54 & 0.293 \\
\hline \multicolumn{8}{|l|}{ Anterior horn } \\
\hline B & -0.08 & -0.03 & -0.04 & -0.05 & 1 & 1 & -0.16 \\
\hline$\beta$ & -0.04 & -0.08 & -0.10 & -0.12 & 0.07 & 0.12 & -0.07 \\
\hline $\mathrm{p}$ Value & 0.346 & 0.068 & 0.023 & 0.007 & 0.149 & 0.014 & 0.008 \\
\hline \multicolumn{8}{|l|}{ Posterior horn } \\
\hline B & 0.00 & -0.02 & -0.04 & -0.06 & 1 & 4 & -0.22 \\
\hline$\beta$ & 0.00 & -0.03 & -0.04 & -0.07 & 0.06 & 0.20 & -0.05 \\
\hline $\mathrm{p}$ Value & 0.983 & 0.450 & 0.359 & 0.105 & 0.179 & $<0.001$ & 0.085 \\
\hline \multicolumn{8}{|c|}{ Periventricular body } \\
\hline B & -0.20 & -0.02 & -0.04 & -0.05 & 1 & 2 & -0.25 \\
\hline$\beta$ & -0.07 & -0.04 & -0.06 & -0.09 & 0.08 & 0.15 & -0.08 \\
\hline$p$ Value & 0.102 & 0.362 & 0.172 & 0.045 & 0.080 & 0.002 & 0.005 \\
\hline \multicolumn{8}{|c|}{ Total brain white matter } \\
\hline B & -1.38 & -0.21 & -0.21 & -0.29 & 3 & 8 & -1.06 \\
\hline$\beta$ & -0.14 & -0.11 & -0.09 & -0.13 & 0.05 & 0.15 & -0.09 \\
\hline $\mathrm{p}$ Value & 0.003 & 0.015 & 0.040 & 0.003 & 0.299 & 0.001 & 0.002 \\
\hline
\end{tabular}

Significant effects $(p<0.01)$ are shown in bold.

$\mathrm{BH}$, both hands; $\mathrm{DH}$, dominant hand; $\mathrm{NDH}$, non-dominant hand.

moving a table, pushing a vacuum cleaner, playing golf, bowling) or vigorous activities (for example, running, lifting heavy weights, participating in strenuous sports), climbing a flight of stairs, bending kneeling or stooping, and bathing or dressing. Subjects with more WMHs were also more likely to report that overall their physical health had prevented them from accomplishing at an optimal level. The items of the SF-12 are scored with factor weights provided by the developers, ${ }^{18}$ such that the physical component scale is uncorrelated with mental health. Furthermore, our regression analyses adjusted for possible depression or cognitive impairment in these subjects, suggesting that the report of poor health cannot be explained on the basis of these potentially confounding factors.

The SF-12 is a self report scale, and it is possible that subjective report of poor physical health may be affected by a reporting bias. However, this is unlikely to be a satisfactory explanation because the same subjects did not also report poor mental health. More importantly, they performed less well on objective tests such as the Purdue Pegboard, choice reaction time, grip strength, and the SDMT, suggesting that they had slowing of fine movement, reduced speed of information processing, and reduced muscle strength. The finding of reduced speed of information processing is consistent with the published literature. ${ }^{21}$ This is explained on the basis of the disruption of widely distributed neural networks by the lesions represented by WMHs. ${ }^{22}$

Findings of pure motor abnormalities in asymptomatic subjects with WMHs have been less consistent. Gait and balance abnormalities are frequently related to leukoaraiosis, dating back to the classic description by Binswanger. ${ }^{23}$ Many recent studies have reported that in elderly individuals, increased WMHs are associated with gait dysfunction and reduced mobility. ${ }^{34-26}$ We did not make an assessment of gait and balance in our study. In contrast, fine motor performance has not generally been associated with WMHs. GunningDixon and Raz ${ }^{2}$ reviewed seven studies and found a low and non-significant correlation $(r=0.09)$ between WMHs and motor performance. The median sample size of these studies was only 41, however. Many other studies assessing information processing speed failed to include tests of pure motor performance. A recent study of normal older individuals, with a mean age of 78.5 years $^{27}$ and a sample size of 59 , also reported no significant relation between Pegboard scores and WMHs. Our study is one of the first to show an association between WMHs and motor tasks. The Purdue Pegboard test was developed to assess manual dexterity, ${ }^{19}$ and may be regarded as a pure motor task, as is the grip strength assessed with a hand dynamometer. ${ }^{20}$ Importantly, the subjects of our study were in their early 60s and were randomly selected from the community. Although the WMHs were present in all subjects, they were small in extent, affecting a mean $0.83 \%$ of the white matter. Therefore, the finding of poor physical health and motor abnormalities in this sample is particularly noteworthy.

We think that there are two possible explanations for the association of WMHs with poor physical health, namely: (1) WMHs lead to the development of physical impairment, 
which is subtle but is recognised by the subjects; and (2) both WMHs and poor physical health are related to some other common factors but do not have a direct causal link. Although age is one common factor, it was not a significant determinant in our study, probably because of the narrow age range studied. We considered the possibility that known risk factors for WMHs, such as hypertension and diabetes, associated with small vessel disease, may also be related to poor physical health, but did not find a significant association of either of these with the SF-12 score. In contrast, the Purdue Pegboard score and grip strength were significantly related to the SF-12 score, suggesting that motor abnormality may mediate an effect of WMHs on physical disability. It is likely that both the pyramidal and extrapyramidal systems are involved by the WMHs, but this cannot be confidently resolved from their topographical distribution. The progression of physical disability in the future may help to determine the mechanism of physical disability.

The association with motor abnormality was stronger for DWMHs than PVHs, even though we found some significant associations for both locations. For the DWMHs, there was no specific association with a particular lobar region, except that the occipital lobe WMHs were not significantly related to physical health and the frontal and parietal regions were. Previous reports on the location of WMHs and functional abnormality have been inconsistent, and many studies have failed to examine this issue. ${ }^{21}$ The stronger relation seen for DWMHs is consistent with the importance of subcortical white matter in some of the neuronal circuits involved in motor function.

A surprise finding of our study, which is not the main focus of this paper, was the higher density of WMHs in women, both in the periventricular region and in deep white matter. Women had lower rates of hypertension, diabetes, and cardiac disease, which are recognised risk factors for cerebrovascular disease. It is possible that this sex difference is an artefact of the sampling process owing to the high refusal rate, even though there were no sex differences in the refusal rates at the various stages of subject recruitment. The sex differences in WMHs have received little attention to date. This finding raises the question of whether we adequately understand the aetiopathogenesis of WMHs, especially in women. We speculate that genetic and/or hormonal factors interact with vascular risk factors in producing differential effects on white matter in men and women. Women did show greater disability on the SF-12, in line with the higher load of WMHs. They also reported more depressive symptoms.

We did not find a significant relation between WMHs and cognitive functions such as episodic memory, working memory, and general intellectual functioning. This is in contrast to the previous literature in which WMHs have been found to be associated with frontal executive function, memory, and global cognitive functioning. ${ }^{21} 28$ However, we did not conduct a detailed assessment of frontal executive function. Our test battery did include the CVLT, a well established test for memory, and Spot the Word test for general intellectual functioning. The negative finding in our sample may be explained on the basis that ours was a relatively young sample compared with previous studies, and the association may only be apparent in an older group with a greater severity of WMHs. It must also be pointed out that our method of measuring of WMHs was automated, offering greater reliability, but having the disadvantage of being sensitive to very mild lesions compared with visual rating methods.

A limitation of our study is the cross sectional design, which prevents causal inferences to be drawn. This sample will be re-examined in 2005 for progression of WMHs and the change in physical disability. Its strength is in the large sample size that was drawn randomly from the community. The suggestion that individuals in their early 60s may suffer physical impairment as a result of lesions in their white matter deserves further study. Because some risk factors for WMHs have been identified, attention to these should begin early in life. We need further research into factors that may be protective against such lesions, so that effective preventative strategies can be put into place.

\section{ACKNOWLEDGEMENTS}

This study was supported by Project Grant ID 157125 and Program Grant 179805 from the National Health and Medical Research Council (NHMRC) of Australia. We wish to thank the following for their contribution to the PATH Through Life Project: K Anstey, J Cullen, T Jacomb, R Kumar, J Maller, K Maxwell, C Meslin, J Price FRACR, B Rodgers, and the PATH Interviewing Team. A Russell prepared the manuscript.

\section{Authors' affiliations}

P S Sachdev, W Wen, School of Psychiatry, University of New South Wales, Sydney, NSW 2052, Australia

H Christensen, A F Jorm, Centre for Mental Health Research, Australian National University, Canberra, 0200, Australia

Competing interests: none declared

\section{REFERENCES}

1 Longstreth WT Jr, Manolio TA, Arnold A, et al. Clinical correlates of white matter findings on cranial magnetic resonance imaging of 3301 elderly people. Stroke 1996;27:1274-82.

2 Gunning-Dixon FM, Raz N. The cognitive correlates of white matter abnormalities in normal aging: a quantitative review. Neuropsychology 2000; 14:224-32.

3 Starr JM, Leaper SA, Murray AD, et al. Brain white matter lesions detected by magnetic resonance imaging are associated with balance and gait speed. J Neurol Neurosurg Psychiatry 2003;74:94-8.

4 Sakakibara R, Hattori T, Uchiyama T, et al. Urinary function in elderly people with and without leukoaraiosis: relation to cognitive and gait function. I Neurol Neurosurg Psychiatry 1999;67:658-60

5 Steffens DC, Bosworth HB, Provenzale JM, et al. Subcortical white matter lesions and functional impairment in geriatric depression. Depress Anxiety 2002;15:23-8.

6 Pantoni L, Garcia JH. Pathogenesis of leukoaraiosis: a review. Stroke 1997;28:652-9.

7 Schmidt R, Fazekas F, Kleinert G, et al. Magnetic resonance imaging signal hyperintensities in the deep and subcortical white matter: a comparative study between stroke patients and normal volunteers. Arch Neurol 1992;49:825-7.

8 Wen W, Sachdev PS. The topography of white matter hyperintensities on brain MRI in middle-aged individuals. Neuroimage 2004;22:144-54.

9 Fazekas F, Chawluk JB, Alavi A, et al. MR signal abnormalities at $1.5 \mathrm{~T}$ in Alzheimer's dementia and normal aging. Am J Neuroradiol 1987; 149:351-6.

10 Duvernoy $\mathrm{H}$. The human brain: surface, three-dimensional sectional anatomy and MRI. Wein/New York: Springer-Verlag.

11 Jorm AF, Anstey KJ, Christensen H, et al. Gender differences in cognitive abilities: the mediating role of health state and health habits. Intelligence 2004;32:7-23

12 Wechsler D. Wechsler memory scale-revised manual. San Antonio, Texas: The Psychological Corporation, 1991.

13 Smith A. Symbol digit modalities test (SDMT) manual. Los Angeles: Western Psychological Services, 1982.

14 Delis DC, Kramer JH, Kaplan E, et al. California verbal learning test. San Antonio: Psychological Corporation Harcourt Brace Jovanovich, 1987.

15 Baddeley A, Emslie H, Nimmo-Smith I. The spot-the-word test. Bury St Edmunds, UK: Thames Valley Test Company, 1992.

16 Folstein MF, Folstein SE, McHugh PR. "Mini-Mental State': a practical method for grading the cognitive status of patients for the children. J Psychiatr Res 1975; 12:189-98.

17 Spitzer RL, Kroenke K, Williams JBW. Validation and utility of a self-report version of PRIME-MD: the PHG primary care study. JAMA 1999;282:1737-44.

18 Ware JE, Kosinski M, Keller SD. A 12-item short-form health survey: construction of scales and preliminary tests of reliability and validity. Med Care 1996;34:220-33.

19 Tiffin J. Purdue pegboard examiner's manual. Rosemont, IL: London House, 1968.

20 Reitan RM, Wolfson D. The Halstead-Reitan neuropsychological test battery: theory and clinical interpretation. Tucson, Arizona: Neuropsychological Press, 1993.

21 de Groot JC, de Leeuw FE, Oudkerk M, et al. Cerebral white matter lesions and cognitive function: the Rotterdam scan study. Ann Neurol 2000;47:145-51. 
22 Filley CM. The behavioral neurology of cerebral white matter. Neurology 1998;50:1535-40.

23 Thompson PD, Marsden CD. Gait disorder of subcortical arteriosclerotic encephalopathy: Binswanger's disease. Mov Disord 1987;2:1-8.

24 Whitman GT, Tang Y, Lin A, et al. A prospective study of cerebral white matter abnormalities in older people with gait dysfunction. Neurology 2001;57:990-4.
25 Guttman CR, Benson R, Warfield SK, et al. White matter abnormalities in mobility-impaired older persons. Neurology 2000;54:1277-83.

26 Baloh RW, Yue Q, Socotch TM, et al. White matter lesions and disequilibrium in older people. I. Case-control comparison. Arch Neurol 1995:52:970-4.

27 Baloh RW, Ying SH, Jacobson KM. A longitudinal study of gait and balance dysfunction in normal older people. Arch Neurol 2003:60:835-9.

28 de Groot JC, de Leeuw FE, Oudkerk M, et al. Cerebral white matter lesions and subjective cognitive dysfunction. Neurology 2001;56:1539-45.

\section{NEUROLOGICAL PICTURE}

Giant calcified chronic subdural haematoma: a long term complication of shunted hydrocephalus

A 15 year old boy, who had undergone ventriculoperitoneal (VP) shunting for hydrocephalus at age 5, presented with a several-month history of progressive right hemiparesis, headache, and urinary incontinence for 3 weeks. Clinical examination revealed grade 3 power (MRC classification) in the right limbs. Cranial computed tomography (CT) showed a high density mass with surface calcification (fig 1A-D).

Preoperatively it was considered he had a chronic, calcified subdural haematoma from VP shunt overdrainage. The mass was removed via craniotomy and confirmed organised subdural blood clot, which was thickly calcified on its surface. Seven days post operation, CT revealed that the haematoma had been totally resected, the brain had expanded, and the lateral ventricles were large (fig 2A-D).

One month later his preoperative motor symptoms had resolved. Over the next year his mental status improved and he became capable of all activities of daily living despite persistent hydrocephalus on serial CT scans. He was considered to have compensated chronic hydrocephalus.

Calcification of chronic subdural haematomas, which are caused by over drainage of cerebrospinal fluid (CSF) by shunting, ${ }^{1}$ is not common. Several reported cases have been asymptomatic, implying that CSF is shunted enough to maintain normal intracranial pressure. Bilateral calcified
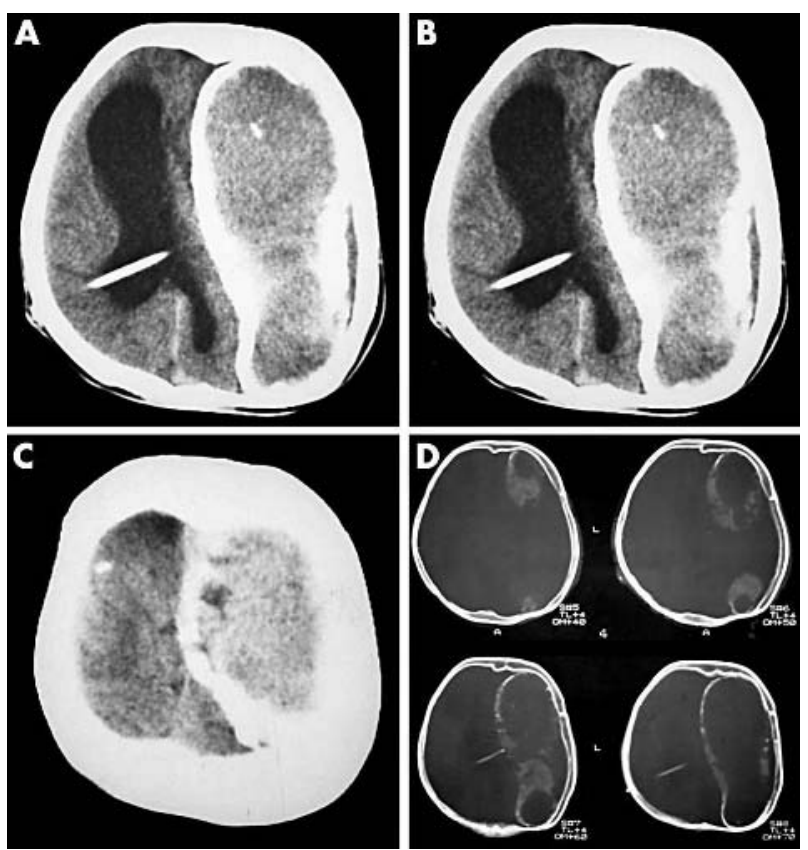

Figure 1 (A-D) Preoperative cranial CT. Cranial CT scans revealed a higher density mass with significant calcification around its surface, occupying larger intracranial space.
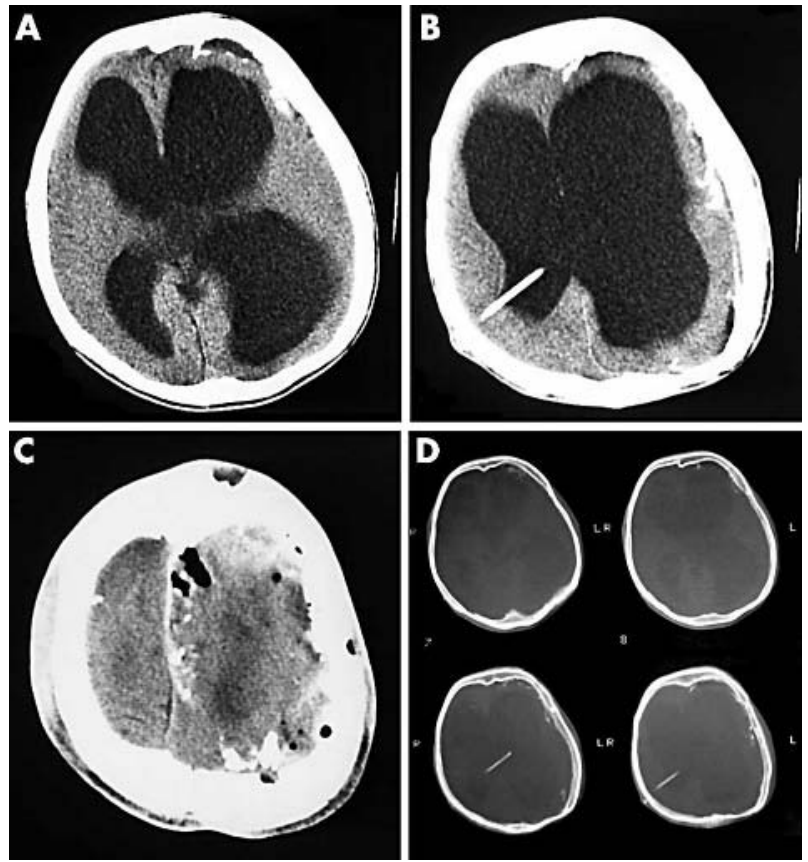

Figure 2 (A-D) Postoperative cranial CT. At 7th day post operation cranial CT scans manifested that calcified and organised subdural haematoma was totally removed, and CSF was accumulated in lateral ventricles and expanded outwards cerebral parenchyma that occupied the residual space at which the subdural haematoma had been located.

subdural haematomas-"armoured brain"—can, however, result in severe symptoms..$^{3-5}$ Excision of the haematoma can-as in this patient-lead to good outcome.

X S He, X Zhang

Department of Neurosurgery, Xijing Hospital, China

Correspondence to: Dr He Xiaosheng, Department of Neurosurgery, Xiiing Hospital, Fourth Military Medical University, Xi'an, Shaanxi 710032, China; hexiaos@fmmu.edu.cn

\section{References}

1 Tuli S, Drake J, Lawless J, et al. Risk factors for repeated cerebrospinal shunt failures in pediatric patients with hydrocephalus. $J$ Neurosurg 2000;92(1):31-8

2 Barmeir EP, Stern D, Harel S, et al. Calcified subdural hematomas associated with arrested hydrocephalus--late sequelae of shunt operation in infancy. Eur J Radiol 1985;5(3):186-9.

3 Spadaro A, Rotondo M, Di Celmo D, et al. Bilateral calcified chronic subdural hematoma. Further pathogenetic and clinical consideration on the so-called "armored brain". J Neurosurg Sci 1987;31(2):49-52.

4 Sharma RR, Mahapatra A, Pawar SJ, et al. Symptomatic calcified subdural hematomas. Pediatr Neurosurg 1999;31:150-4.

5 Ersahi Y, Mutluer S. Calcified subdural hematoma. Pediatr Neurosurg 2000;32:165-6. 